Cahiers d'études italiennes

Enea Silvio Piccolomini-Pie II

\title{
L'éducation du prince dans le Tractatus de liberorum educatione
}

Cécile Terreaux-Scotto

\section{OpenEdition}

Journals

Édition électronique

URL : http://journals.openedition.org/cei/79

DOI : $10.4000 /$ cei. 79

ISSN : 2260-779X

\section{Éditeur}

UGA Éditions/Université Grenoble Alpes

\section{Édition imprimée}

Date de publication : 15 octobre 2011

Pagination : 103-128

ISBN : 978-2-84310-207-3

ISSN : $1770-9571$

\section{Référence électronique}

Cécile Terreaux-Scotto, "L'éducation du prince dans le Tractatus de liberorum educatione », Cahiers d'études italiennes [En ligne], 13 | 2011, mis en ligne le 15 avril 2013, consulté le 27 mars 2021. URL http://journals.openedition.org/cei/79 ; DOI : https://doi.org/10.4000/cei.79 


\title{
L'ÉDUCATION DU PRINCE DANS LE TRACTATUS DE LIBERORUM EDUCATIONE
}

\author{
Cécile Terreaux-Scotto \\ Université Stendhal - Grenoble 3
}

En I450, à l'occasion de Noël, l'évêque de Trieste Enea Silvio Piccolomini, qui allait accéder au trône pontifical huit ans plus tard sous le nom de Pie II, rédige une très longue lettre à l'intention du jeune Ladislas le Posthume, duc d'Autriche et héritier de la couronne de Bohème et de Hongrie ${ }^{\mathrm{I}}$. Le jeune prince, âgé d'une dizaine d'années, se trouvait alors sous la tutelle de l'empereur Frédéric III $^{2}$. Bien que Piccolomini n'ait jamais enseigné, il établit dans ce texte un programme éducatif complet, qui s'appuie en très grande partie sur le De institutione oratoria de Quintilien que Poggio Bracciolini avait entièrement publié en I4I6 et sur le traité pédagogique de Plutarque que Guarino Veronese avait traduit à Florence en I4II ${ }^{3}$. Se préoccupant à la fois du corps et de l'esprit des enfants à former, Piccolomini accorde une place prédominante à la littérature et à la philosophie, sources de sagesse pour qui prétend gouverner un peuple.

En 1958, Eugenio Garin a traduit en italien cette œuvre, connue sous le titre de Tractatus de liberorum educatione, en même temps que d'autres textes traitant de l'éducation des enfants, tous publiés dans le volume Il pensiero pedagogico dell'umanesimo ${ }^{4}$. Craig Kallendorf s'est d'ailleurs appuyé sur ce travail pour établir une traduction anglaise du Tractatus's.

I. Ladislas est né après la mort de son père l'empereur Albert II d'Habsbourg.

2. Il s'agit de Frédéric de Habsbourg, roi des Romains et empereur romain germanique qui en I442 avait conféré à Piccolomini le titre prestigieux de poète.

3. Voir à ce propos A. Van Heck, " Plutarco e l'educazione nell'Umanesimo » dans L'educazione e la formazione intellettuale nell'età dell'Umanesimo (Atti del II Convegno internazionale, I990), Milan, Guerini e Associati, 1992, p. 99-108.

4. E. Garin (éd.), Il pensiero pedagogico dell'umanesimo, Florence, Giuntine-Sansoni, I958. Je citerai d'après cette édition, l'épître de Piccolomini étant désormais désignée par l'abréviation Tractatus.

5. C. W. Kallendorf, Humanist educational treatises, Cambridge / Londres, The I Tatti Renaissance Library / Harvard University Press, 2002, p. I26-259. 
Le traité de Piccolomini, qui se présente comme une longue épître, n’a pas été négligé par la critique. Dès 1960, Del Donno a voulu en faire un commentaire critique; il a toutefois utilisé une traduction tellement tronquée que le texte de Piccolomini en est défiguré à un point tel que même les commentaires deviennent pratiquement inutilisables ${ }^{6}$. CharlesÉdouard Naville y a consacré une dizaine de pages dans sa biographie de Piccolomini publiée en 1984, sans toujours se détacher de la paraphrase ${ }^{7}$; quant à Gioacchino Paparelli, le biographe de référence du pape Pie II, il ne fait que de brèves allusions au Tractatus ${ }^{8}$.

En revanche d'autres études font souvent allusion de façon stimulante au texte pédagogique de Piccolomini. C'est le cas d'un article de Lionello Sozzi, qui analyse de nombreux passages du Tractatus pour définir le sens de la dignitas dans la pensée de Piccolomini ${ }^{9}$. En outre de nombreux articles éclairants ont développé des points précis du traité. Ubaldo Pizzani a étudié la place qu'y occupent le quadrivium et le trivium par rapport à la culture médiévale ${ }^{\mathrm{IO}}$. Marco Ballarini et Franco Buzzi ont montré que le texte de Piccolomini se trouve à la croisée des traités d'éducation humanistes et des miroirs des princes ${ }^{\text {II }}$. Manfred Lentzen a dégagé les principales idées du Tractatus en l'associant à deux autres écrits pédagogiques de Piccolomini : l'épître au duc Sigismond d'Autriche rédigée en I443 et une lettre adressée l'année suivante à Guillaume von Stein ${ }^{12}$. Bien plus tôt, Albert Baca avait mis en perspective le Tractatus avec d'autres textes de Piccolomini, ses lettres à Sigismond et au cardinal Olesnicki et son Artis rhetoricae praecepta en particulier ${ }^{13}$. Quant à la lecture récente que Luigi Guerrini propose du traité, en le situant dans son contexte non seulement culturel mais aussi historique, elle éclaire de façon particulièrement convaincante la pensée politique de Piccolomini ${ }^{14}$.

6. M. Del Donno, Enea Silvio Piccolomini. L'educazione dei giovani. L'umanesimo e i suoi problemi educativi, Milan-Bari, Educa Editrice, 1960.

7. C.-E. Naville, Enea Silvio Piccolomini. L’uomo l'umanista il pontefice (I405-I464), Locarno, Analisi, 1984.

8. G. Paparelli, Enea Silvio Piccolomini : l'umanesimo sul soglio di Pietro, Ravenne, Longo, 1978, p. 67-68.

9. L. Sozzi, "La dignitas hominis nel pensiero di Pio II " dans Pio II e la cultura del suo tempo (Atti del I Convegno internazionale, luglio 1989), Milan, Guerini e Associati, 1991, p. 47-57.

Io. U. Pizzani, "Discipline letterarie e discipline scientifiche nel De liberorum educatione di Enea Silvio Piccolomini » dans L. Rotondi Secchi Tarugi (éd.), Pio II e la cultura..., ouvr. cité, p. 313-327.

II. M. Ballarini, F. Buzzi, " La formazione filosofico-politica e letteraria nel De liberorum educatione di Enea Silvio Piccolomini » dans Pio II umanista europeo (Atti del XVII Convegno internazionale, Chianciano-Pienza, I8-2I luglio 2005), Florence, Franco Cesati Editore, 2007, p. 567-582.

I2. M. Lentzen, «Le idee pedagogiche di Enea Silvio Piccolomini » dans Pio II umanista ..., ouvr. cité, p. 583-59I.

13. A. R. Baca, "The Educational Theory of Aenas Silvius Piccolomini " dans Acta convenus neolatini turonensis, Paris, 1980, I, p. 369-376.

I4. L. Guerrini, "Sulla strada della ragione. Cultura, storia e politica nel De liberorum educatione tractatus " dans Un pellegrinaggio secolare. Due studi su E. S. Piccolomini, Rome, Edizioni di storia e letteratura, 2007, p. III-I8o. 
Je voudrais procéder ici à un commentaire des principaux rouages de cette épître destinée à Ladislas pour tenter de discerner d'une part, ce qui relève de la tradition pédagogique humaniste et plus largement de la conception de l'enfant à la Renaissance et d'autre part, ce qui fait la spécificité de Piccolomini.

Une lecture même rapide du Tractatus de liberorum educatione fait immédiatement surgir une particularité. Il est en effet malaisé d'identifier un destinataire précis. Certes, Piccolomini emploie fréquemment la deuxième personne du singulier et le vocatif pour interpeller directement le futur souverain. C'est le cas lorsqu'il lui fait remarquer, par exemple, que son éducation ne dépend désormais plus d'une nourrice puisqu'il a grandi : " [...] tu n'es désormais plus sous le joug de ta nourrice ${ }^{\mathrm{Is}}$. " Mais il peut également parfois s'adresser non pas au prince lui-même mais à son entourage, en le désignant par une périphrase ("ceux qui te dirigent ${ }^{16}$ ") et en formulant le souhait qu'il se comporte d'une façon conforme à ses propres idées pédagogiques. C'est le cas lorsque Piccolomini cherche à imposer sa vision de la diététique : "[...] si tes domestiques sont avisés ils n'apporteront pas de très nombreux plats en ta présence ${ }^{17}$. " Pour guider les précepteurs, Piccolomini passe ainsi, par le biais de l'adjectif possessif à la deuxième personne, par leur employeur, le jeune Ladislas; sans doute s'agit-il aussi bien de préserver la hiérarchie sociale que de ménager la susceptibilité des professeurs. Au début de son traité, Enea Silvio souligne d'ailleurs explicitement sa stratégie discursive en ces termes : «[...] pendant que je te parle et que je te donne des conseils, je parle et je donne des conseils à tous les précepteurs qui prennent soin de toi ${ }^{18}$. "

Cette façon qu'a Piccolomini de présenter son point de vue est significative de deux éléments sur lesquels je commencerai par m'attarder : l'âge de Ladislas, qui a dix ans au moment où Piccolomini rédige le traité pour lui, et son statut, qui est évidemment singulier puisque Ladislas est destiné à gouverner un peuple. Dans un deuxième temps, nous verrons que l'éducation du prince repose sur deux principes emblématiques de l'humanisme : l'imitation et la persuasion. Enfin, le dernier moment de cette étude analysera le contenu de la formation que Piccolomini entend donner au futur souverain. Le langage, du corps ou de l'esprit, y tient une place toute particulière.

15. Tractatus, p. 226 : "[...] tu iam nutricis evasisti ingum."

I6. Ibid., p. 214: "qui te regunt».

17. Ibid., p. 214: "[...] afferent [...] ministri tui, si sapient, coram te non plurimas epulas."

I8. Ibid., p. $204:$ : [...] dum te alloquor teque commoneo, et preceptores tuos qui tui curam gerunt omnes alloquor et commoneo." 
Dans les premières pages de son épître, Piccolomini rappelle que l'éducation d'un enfant doit être entreprise au plus tôt, " dès le début de l'enfance $^{19}$ ". Comme on le sait, il s'agit là d'un lieu commun développé par les humanistes ${ }^{20}$, qui reprennent eux-mêmes les observations des auteurs grecs et latins. Aristote et Plutarque ont insisté sur ce point ${ }^{21}$, mais c'est le Virgile des Géorgiques, attentif à la plantation des jeunes plants de vigne, que cite Piccolomini lorsqu'il rappelle qu' « il est important de prendre des habitudes à l'âge tendre ${ }^{22}$ ".

Cependant, cette période où il faut prendre garde aux fondations de la personnalité est pour Ladislas définitivement révolue, comme le souligne Piccolomini, «[...] ces temps sont derrière toi ${ }^{23}$ ». Or, cette précision n’est pas sans importance lorsqu'on connaît la façon dont les contemporains d'Enea Silvio envisagent les âges de la vie.

Le premier âge, appelé infantia, n'est en effet pas considéré par les pédagogues humanistes comme l'âge de la formation. Certes, l'individu, comme on vient de le voir, peut et doit être éduqué au plus vite après sa naissance. Mais l'infans est un être qui, à cause de sa dentition inachevée, ne parle pas. Dans l'incapacité de tenir un discours cohérent et articulé, qui puisse être communiqué et compris, l'infans est avant tout un être privé de raison ${ }^{24}$. C'est pourquoi il peut recevoir les bonnes habitudes qui lui sont inculquées au sujet des bonnes manières ou de l'hygiène par exemple, mais il n'est pas en mesure de tirer profit d'une instruction qui ferait appel à des compétences intellectuelles qu'il n'a pas.

En revanche, l'entrée dans la pueritia, qui coïncide traditionnellement avec le septième anniversaire de l'enfant, marque une double évolution. D'un point de vue intellectuel, selon la conception de Varron et d'Isidore

19. Ibid., p. $204:$ : [...] ab infantia et primis. "

20. E. Garin, "L'image de l'enfant dans les traités de pédagogie du Xv siècle » dans E. Becchi et D. Julia, Histoire de l'enfance en Occident. De l'Antiquité au XVIT siècle, Paris, Seuil, I998, t. I, p. 23I.

2I. Aristote, Éthique à Nicomaque, J. Tricot (éd. et trad.), Paris, Vrin, 1997, II, I, p. 90 : "Ce n'est pas une œuvre négligeable de contracter dès la plus tendre enfance telle ou telle habitude, c'est au contraire d'une importance majeure, disons mieux totale. " - Plutarque, De l'éducation des enfants dans Euvres morales, J. Sirinelli (éd. et trad.), Paris, Les Belles Lettres, 1987, t. I, p. 39 : «De même que dès leur naissance il est indispensable de façonner les membres des enfants pour qu'ils se développent bien droits et sans difficulté, de la même manière il convient de régler leurs mœurs dès le départ. "

22. "Multum est in teneris consuescere", Tractatus, p. 230. Par rapport à la citation originale, Piccolomini a simplement antéposé le verbe : "in teneris consuescere multum est! ", écrivait Virgile (Géorgiques, éd et trad. de E. de Saint-Denis, Paris, Les Belles Lettres, 1960, II, v. 272).

23. Tractatus, p. $204:$ : [...] preterita sunt hec tempora tibi."

24. D. Lett, "Aetas infirma, ætas infima ", Médiévales. Le premier Moyen Âge, n 15, 1988, p. 93-94; D. Lett, L'enfant des miracles. Enfance et société au Moyen Âge (XII'-XIII siècles), Paris, Aubier, 1997, p. 28 ; S. Nagel, S. Vecchio, "Il bambino, la parola, il silenzio nella cultura medievale ", Quaderni Storici, n 57, décembre I984, XIX, fasc. 3, p. 719-73I. 
de Séville, communément reprise par les humanistes, la faculté de donner du sens aux mots prononcés, qui cessent alors d'être de simples sons, s'acquiert à l'âge de sept ans. Et d'un point de vue moral, c'est aussi à l'âge de la pueritia que l'enfant sait reconnaitre le bien du mal, lorsqu'il atteint l'atas discretionnis ${ }^{25}$.

Âgé d'une dizaine d'années, Ladislas possède donc ces capacités d'expression et de discernement depuis trois ans. Aussi n'est-ce pas un hasard si Piccolomini tient à souligner que le jeune prince est un puer - "nous te traitons encore comme un enfant "- et que ce sont donc les préceptes adaptés à un puer qui lui sont destinés : "Écoute les préceptes que nous donnons à un enfant ${ }^{26}$."

Sur les traces de Quintilien ${ }^{27}$, Piccolomini veille d'ailleurs attentivement à respecter l'âge de Ladislas, puisqu'il recommande aux précepteurs $\mathrm{du}$ futur roi de ne pas lui faire étudier les textes des théologiens, davantage adaptés "à un autre moment " de sa vie parce qu'ils ont à voir avec la philosophie ${ }^{28}$. Or, si la philosophie est le couronnement de toute formation bien menée, elle est trop difficile pour être maîtrisée par un enfant qui se trouve encore dans la pueritia. Le puer qu'est Ladislas devra donc se contenter dans un premier temps de bases culturelles solides qui lui permettront, mais plus tard justement, d'aborder avec efficacité et sérénité la philosophie.

Il est une autre catégorie de textes que le futur pape écarte, en tout cas provisoirement, du programme éducatif destiné à Ladislas. Il s'agit des poésies élégiaques de Tibulle, Catulle, Properce et Sappho, dont la composante érotique ne sied guère à un puer, et qui conviendront davantage, dit-il, "à un âge plus solide 29 ». Semblablement, Piccolomini évite lui-même de parler d'amour et de sexualité parce que ce sont des discours plus appropriés à un "adolescent " (adolescens) qu’à un " enfant » (puer) : «[...] il faudra informer des choses de l'amour un adolescent et non un enfant ${ }^{30}$."

25. D. Lett, "Aetas infirma... ", art. cité, p. 90; D. Lett, L'enfant des miracles..., ouvr. cité, p. 29.

26. Tractatus, p. 204 : "Adhuc te puerum habemus; audi precepta que damus puero."

27. Quintilien, Institution oratoire, J. Cousin (éd. et trad.), Paris, Les Belles Lettres, 1975, t. I, I, 2, $28:$ « [...] il faut considérer ce que l'esprit des enfants est susceptible d'absorber : car ce qui dépasse leur intelligence ne pénétrera pas dans des esprits trop peu ouverts, pour ainsi dire, pour le recevoir. " (" [...] animi puerorum quantum excipere possint uidendum est : nam maiora intellectu uelut parum apertos ad percipiendum animos non subibunt.")

28. Ibid., p. $266:$ : in aliud tempus».

29. Ibid., p. 268 : «ad firmius etatis robur». Ces recommandations sont déjà présentes chez Quintilien, Institution oratoire..., ouvr. cité, t. I, I, 8, 6.

30. Ibid., p. 2I4: "[...] de Venere adolescentem magis quam puerum commonefacere oportebit. " 
Remarquons à ce propos que c'est la sexualité qui marquera l'entrée de Ladislas dans un autre âge de la vie que la pueritia, à savoir l'adolescentia.

Cette première analyse du Tractatus de liberorum educatione montre que Piccolomini distingue implicitement trois phases dans l'âge de la croissance : l'infantia de la naissance à sept ans, puis la pueritia de sept à quatorze ans, enfin l'adolescentia jusqu'à vingt-quatre ans. Ce schéma, qu'on trouve déjà chez Hippocrate ${ }^{3 \mathrm{I}}$, a été développé au VII ${ }^{\mathrm{e}}$ siècle par le chrétien Isidore de Séville, qui a répandu la vision augustinienne établissant un parallélisme entre les âges de la vie et les six âges du monde ${ }^{32}$. Cette répartition des âges a également été adoptée par un siennois, Aldebrandin de Sienne, médecin de la maison de Savoie au XIII ${ }^{\mathrm{e}}$ siècle, dont le texte rédigé en français a été traduit en italien en Izıo par le notaire Zucchero Benvicenni ${ }^{33}$. Piccolomini s'appuie donc sur ces différentes sources pour construire son discours pédagogique ${ }^{34}$.

Le destinataire de ce discours se trouve justement à un âge, la pueritia, que les pédagogues considèrent comme l'âge de la formation par excellence ${ }^{35}$. Aux XIV et XV siècles, cette conception trouve son expression dans l'image très courante de l'enfant semblable à un morceau de cire que l'on peut modeler facilement ${ }^{36}$. De son côté Piccolomini compare les enfants à un récipient qui conserverait longtemps l'odeur des contenus qu'on y a versés. Il reprend là les mots biens connus qu'Horace adressait lui aussi à un puer qu'il conseillait : «[...] le vase conserve longtemps le parfum

3I. H.-I. Marrou, Histoire de l'éducation dans l'Antiquité, Paris, Seuil, I948, p. I48.

32. Isidori Hispalensis episcopi, Etymologiarum sive originum, W. M. Lindsay (éd.), Oxford, Oxford University Press, 1957, vol. 2, XI, 2, I-8.

33. L. Landouzy, R. Pepin, Le régime du corps de maître Aldebrandin de Sienne, Genève, Slatkine Reprints, I978, p. 79.

34. Sur les âges de la vie, E. Sears, The Ages of Man. Medieval Interpretations of the Life Cycle, Princeton, Princeton University Press, 1986 ; J. A. Burrow, The Ages of Man: A Study in Medieval Writing and Thought, Oxford, Clarendon Press, 1986 ; M. Goodich, From Birth to Old Age. The Human Life Cycle in Medieval Thought, I250-I350, Lanham / New-York / Londres, University Press of America, 1989; S. Shulamith, Childhood in the Middle Ages, Londres / New York, Routledge, 1990, en particulier p. 2I-3I. Les âges de la vie au Moyen Âge (actes du colloque du Département d'Études Médiévales de l'université Paris-Sorbonne et de l'université Friedrich-Wilhem de Bonn, Provins, I6-I7 mars 1990), Paris, Presses de l'université de Paris-Sorbonne, I992 ; D. Chauvin (éd.), L’imaginaire des âges de la vie, Grenoble, Ellug, I996; A. Paravicini Bagliani, "Âges de la vie " dans J. Le Goff et J.-C. Schmitt (éd.), Dictionnaire raisonné de l'Occident médiéval, s. 1., Fayard, I999, p. 7-I9. On trouvera une synthèse récente dans I. Taddei, Fanciulli e giovani. Crescere a Firenze nel Rinascimento, Florence, Olschki, 200I, p. 13-63.

35. A. Giallongo, Il bambino medievale. Educazione ed infanzia nel Medioevo, Bari, Edizioni Dedalo, I990, p. 170.

36. Francesco da Barberino, Reggimento e costumi di donna, G. Sansone (éd.), Turin, Loescher-Chiantore, 1957, XIII, p. 286; G. Dominici, Regola del governo di cura familiare, D. Salvi (éd.), Florence, Angiolo Garinei, I860, p. I96. Ces formulations sont déjà présentes chez Juvénal et Plutarque par exemple. Juvénal, Satires, P. de Labriolle et F. Villeneuve (éd. et trad.), Paris, Les Belles Lettres, I94I, VII, 237-238; Plutarque, De l'éducation des enfants..., ouvr. cité, p. 39. Voir à ce sujet D. Lett, L'enfant des miracles..., ouvr. cité, p. 57 et p. 62-63. 
de la première liqueur dont il a été rempli ${ }^{37}$. " Enea Silvio mentionne en outre deux raisons à la fois opposées et complémentaires pour justifier l'éducation des pueri : d'une part leur faculté d'apprendre, de retenir et d'imiter les rend plus réceptifs aux vertus (Tractatus, p. 238) et d'autre part, leur intelligence pourrait être gâtée de façon irréversible s'ils n'étaient pas nourris par «d'excellentes choses ${ }^{38}$ ".

Le puer peut donc être formé et instruit. L'âge de Ladislas fait alors du puer qu'il est le destinataire emblématique d'une épître à visée pédagogique.

Etre en formation, le puer n'a pas de statut propre par rapport aux adultes, parce qu'il «n'exerce pas encore de responsabilité sociale 39 ». Infirme par nature, il vit en marge de la société, comme l'ont montré les travaux de Richard Trexler sur la société florentine à la Renaissance ${ }^{40}$.

Mais Ladislas n'est justement pas un enfant comme les autres. Les tout premiers mots de Piccolomini, au vocatif, le proclament d'ailleurs avec force. L'épître s'ouvre en effet sur l'apostrophe " ô Ladislas, illustre roi ${ }^{4 \mathrm{I}}$ » : Ladislas est un prince, un "rex puer ", selon la formulation employée de façon récurrente par Piccolomini ${ }^{42}$. Voilà pourquoi il sera éduqué par des précepteurs et non à l'école, contrairement aux autres enfants qui commencent à la fréquenter précisément à l'âge de sept ans lorsqu'ils cessent d'être des infantes ${ }^{43}$.

Le destinataire du De liberorum educatione se distingue donc d'emblée, par sa naissance et l'éducation qui lui est liée, des autres pueri. Le passage où Piccolomini mentionne toutes les activités nécessaires à un futur soldat est particulièrement significatif à cet égard.

Pour préparer Ladislas au combat, Piccolomini recommande la pratique du tir à l'arc, de la fronde, du lancer, de l'équitation, de la course, du saut, de la chasse et de la nage ${ }^{44}$. Comme on le sait, ce sont là des activités habituellement recommandées par les pédagogues humanistes, qui s’inscrivent dans la lignée des préceptes antiques, à commencer par

37. Tractatus, p. $266:$ : [...] quo semel est imbuta recens servabit odorem testa diu. " La citation d'Horace est calquée mot pour mot. Voir Horace, Épîtres, F. Villeneuve (éd. et trad.), Paris, Les Belles Lettres, I955, I, 2, 69-70.

38. Tractatus, p. 270-272: "optimis rebus".

39. J.-C. Payen, "L'enfance occultée : note sur un problème de typologie littéraire au Moyen Âge ", Sénéfiance, L'enfant au Moyen Âge, n 9, 1980, p. I86.

40. R. Trexler, Public Life in Renaissance Florence, Ithaca / Londres, Cornell University Press, I980, p. I5-I6.

4I. Tractatus, p. 198 : "Ladislae, rex inclite."

42. Tractatus, p. 2 II, p. 272 , p. 287 , p. 288 , p. 290.

43. Sur cette question, C. Klapisch-Zuber, "Les clefs florentines de Barbe-Bleue " dans La maison et le nom. Stratégies et rituels dans l'Italie de la Renaissance, Paris, EHESS, I990, p. 309-330; D. Herlihy, C. Klapisch-Zuber, Les Toscans et leurs familles. Une étude du catasto florentin de I427, Paris, EHESS, I978, p. 563-568.

44. Tractatus, p. 210: " [...] arcum in pueritia tendere, sagittam dirigere, fundam rotare, hastam iacere, equos ascendere, cursitare, saltare, interesse venationibus, nancisci nandi peritiam. " 
ceux de Plutarque ${ }^{45}$. À la Renaissance, Leon Battista Alberti, pour ne citer que lui, préconise exactement les mêmes exercices que Piccolomini : que les jeunes gens " tirent à l'arc " et " montent à cheval " et qu'ils apprennent aussi à "faire de l'escrime " et à " nager " ${ }^{46}$. Pour Alberti comme pour Piccolomini, il s'agit de former les jeunes gens à la guerre. C'est que, dit Alberti, l'entraînement est nécessaire en prévision d'une hypothétique situation de conflit militaire, " pour pouvoir au besoin être utile contre les ennemis de la patrie ${ }^{47}$ ». Mais chez Piccolomini l'hypothèse d'Alberti est prévision, voire prévoyance, parce qu'il ne fait aucun doute pour le futur pape que Ladislas combattra un jour, et même qu'il se battra contre les Turcs : «toi, qui devras souvent combattre les Turcs " ${ }^{48}$, le prévient-il. Cet avertissement anticipe au passage le souhait exprimé quelques années plus tard par Pie II de voir une croisade menée contre les Turcs ${ }^{49}$.

Cette perspective éclaire en tout cas les préoccupations diététiques de Piccolomini. Selon lui, l'alimentation du jeune prince doit en effet être pensée en fonction de son avenir guerrier. Il est nécessaire que Ladislas s'habitue à une nourriture ordinaire, non seulement parce qu'il lui faut fortifier son corps, mais aussi parce qu'il lui arrivera d'être en campagne, où il pourra tout manger, y compris de la viande de boeuf (Tractatus, p. 2I2)! Ainsi, là où Alberti cherche à préparer les jeunes gens à une situation éventuelle, Piccolomini trace pour Ladislas le destin d'un prince-soldat; à la possibilité pour un jeune aristocrate d'être confronté à la guerre répond la certitude du devoir de croisade pour un roi chrétien.

Par conséquent, même si pour l'instant le puer Ladislas est sous la tutelle de Frédéric III, il a déjà, malgré son jeune âge, un statut dans la société, celui d'héritier d'un trône royal. Piccolomini le souligne du reste dès les premières phrases de son épître : plus qu'un autre, Ladislas a besoin d'acquérir "vertu " et "sagesse " parce qu'il est appelé à gouverner plus tard.

Or, ce statut va de pair avec une responsabilité importante : Ladislas doit se montrer digne, aux yeux de tous, de ses ancêtres. Car les gènes de la noblesse, remarque Piccolomini, ne suffisent pas à déterminer la vertu d'un individu; encore faut-il que le prince héritier soit lui-même vertueux : "Qui hérite du règne de ses ancêtres doit aussi hériter de leurs vertus ${ }^{\circ 0}$."

45. Plutarque, De l'éducation des enfants..., ouvr. cité, p. 50.

46. L. B. Alberti, I libri della famiglia, R. Romano, A. Tenenti (éd.), Turin, Einaudi, 1969, p. 87 et 88 : « saettino", " cavalchino", "schermire ", "notare".

47. Ibid., p. 88 : "[...] per potere al bisogno essere contro gl'inimici alla patria utili.»

48. Tractatus, p. $210:$ : [...] te, quem sepe contra Turcos pugnare necessum erit."

49. Sur ce point, voir G. Paparelli, Enea Silvio Piccolomini..., ouvr. cité, p. 230-262.

50. Tractatus, p. 198-200: "Maiorum qui regna suscipit, ut virtutes quoque suscipiat consentaneum est. " 


\section{Déjà Cicéron affirmait dans le De Officiis que}

le meilleur héritage que transmettent les parents à leurs enfants et qui l'emporte sur tout patrimoine, c'est la gloire de leur vertu et de leurs entreprises : la déshonorer doit être jugé comme un sacrilège et une tare ${ }^{\text {II }}$.

Cette mise en garde est reprise de façon récurrente par les contemporains de Piccolomini. Matteo Palmieri prévient que "celui qui détruit la réputation de ses ancêtres est misérable ${ }^{52}$ »; Pier Paolo Vergerio voit à son tour une faute grave dans le fait de ne pas se montrer à la hauteur de ses aïeux : «[...] il est honteux que nos mœurs et notre vie dévient de leur trajectoire vertueuse ${ }^{53}$. " Piccolomini formule un avertissement semblable lorsqu'il observe que Ladislas bénéficie des préceptes remarquables des générations d'empereurs romains qui l'ont précédé et que ce serait une faute que de les trahir : «[...] ce serait une très grande honte de dévier de l'exemple de tes aïeux [...], de tes ancêtres paternels et maternels et de celui qui t'engendra ${ }^{54}$."

Ladislas est ainsi appelé à un devoir de répétition : marcher sur les traces de ses aïeux afin de ne pas être infidèle à leur vertu et à leur mémoire. Ses maîtres feront donc en sorte que le jeune prince agisse conformément aux qualités qu'il a reçues à la naissance. Car si Ladislas bénéficie d'une position sociale prestigieuse et vertueuse, il lui appartient de faire fructifier son héritage. Sans éducation, la vertu que le prince a reçue à sa naissance demeurerait à l'état de virtualité; seule la formation qu'il recevra la transformera en réalité. Quelle est cette formation que Piccolomini souhaite voir dispensée à Ladislas?

À la suite d'Aristote et de Plutarquess, Piccolomini rappelle que trois éléments sont indispensables à l'éducation d'un enfant : la " nature ", le «savoir » et l'« exercice» (Tractatus, p. 204). La «natura » représente l'inné, c'est-à-dire les facultés que l'individu reçoit à la naissance. La "disciplina » ou «doctrina» est conférée par l'instruction, qui apporte les connaissances

5I. Cicéron, Les Devoirs, M. Testard (éd. et trad.), Paris, Les Belles Lettres, 1965, I, XXXIII, I2I : " Optima [...] hereditas a patribus traditur liberis omnique patrimonio prostantior, gloria uirtutis rerumque gestarum, cui dedecori esse nefas et uitium iudicandum est. "

52. M. Palmieri, Vita civile, G. Belloni (éd.), Florence, Sansoni, I982, p. I36: «[...] misero è colui che consuma la fama de' padri antichi."

53. E. Garin, Educazione umanistica, Bari, Laterza, 1949, p. I08 : " [...] è turpe tralignare nel costume e nella vita dalla loro virtù."

54. Tractatus, p. 198 : «[...] maiorum tuorum [...] paterni ac materni progenitores, et qui te genuit [...], a quibus degenerare fedissimum fuerit. "

55. Aristote, Les politiques, P. Pellegrin (éd.), Paris, Flammarion, I990, VII, II, I332a ; Plutarque, De l'éducation des enfants..., ouvr. cité, p. 36. 
nécessaires au développement des qualités de l'individu. Enfin, l'« exercitatio " consiste à pratiquer des exercices destinés à activer ces connaissances. La " disciplina " et l' " exercitatio " sont donc de l'ordre de l'acquis. Piccolomini ne sépare cependant pas l'inné de l'acquis. Au contraire, reprenant une fois de plus une formulation de Plutarque ${ }^{56}$, il considère que le don naturel, l'apprentissage et l'exercice régulier constituent trois éléments complémentaires et indissociables agissant de façon réciproque les uns sur les autres, et que seule la combinaison de leurs actions peut conduire l'individu à la perfection :

De la même façon que la nature sans le savoir est aveugle, le savoir sans la nature est mutilé. Aucun des deux n'a d'importance sans l'exercice, mais des trois choses découlent la perfection ${ }^{57}$.

La nature humaine, ce don de Dieu, est de toute façon ainsi faite que rares sont les enfants sur qui l'éducation n'aurait aucune prise; et si c'était le cas, il s'agirait alors, ajoute Piccolomini, d'êtres monstrueux et hors-normes. À l'opposé se trouve justement Ladislas, pourvu d'une nature non seulement docile, mais déjà honnête, puisqu'il fuit les amis dépravés et les choses corrompues en même temps qu'il recherche les bons conseils (Tractatus, p. 200 et p. 202).

Deux choses devront en tout cas être formées chez le jeune prince : le corps et l'esprit. Mais qu'il s'agisse de la cura corporis ou de l'institutio mentis, la formation proposée par Piccolomini s'articule, dans son ensemble, autour de deux axes qui se trouvent au cœur de l'éducation humaniste : l'imitation d'une part, la persuasion d'autre part.

Pour commencer - et il s'agit là encore d'une recommandation présente chez Plutarque ${ }^{58}$ - les précepteurs doivent être choisis non seulement en raison de leur érudition (Tractatus, p. 204) mais également en raison de leur moralité, de façon à ce qu'ils puissent donner un « excellent exemple » à leur élève ${ }^{9}$. Rappelons que la paideia grecque reposait déjà sur cette méthode, qui faisait admirer des " personnages modèles » aux jeunes gens ${ }^{60}$. La société

56. Voici le texte de la traduction latine de Guarino, cité par Garin dans son édition du Tractatus : "Caeca enim res sine disciplina natura et absque natura disciplina manca res existit. Imperfectum autem quiddam existit, si ambobus his careat exercitatio. " On trouve la traduction française suivante dans l'édition des Belles Lettres : "Car la nature sans l'instruction est aveugle, l'instruction, séparée de la nature, insuffisante, et l'exercice, sans les deux premiers éléments, inopérant. " (Plutarque, De l'éducation des enfants..., ouvr. cité, p. 36.)

57. Tractatus, p. 204 : "Sicut enim ceca est sine disciplina natura, sic sine natura manca est disciplina. In utraque parum momenti fuerit, si subtraxeris exercitium, sed hisce tribus absolutio comparatur. "

58. Plutarque, De l'éducation des enfants..., ouvr. cité, p. 40.

59. Tractatus, p. 206 : "optimum experimentum».

6o. W. Jaeger, Paideia. La formation de l'homme grec, Paris, Gallimard, I964, p. IX. 
romaine faisait de même, insistant sur l'utilité des exemples fournis par les parents et les ancêtres de l'enfant ${ }^{61}$.

Dans les textes du Moyen Âge et de la Renaissance, c'est la figure du père qui prend une importance fondamentale - dans la famille, il est en effet le seul et unique responsable de l'éducation de l'enfant ${ }^{62}$. "Nous voyons que tout fils regarde davantage les traces des pieds de son père que celles d'autrui ", écrivait Dante, qui souhaitait qu'un père devant son fils " prenne garde de ne pas donner dans ses actions un exemple de luimême qui soit contraire aux exigences de la perfection ${ }^{63}$ ». Plus tard, à la même époque que Piccolomini, Alberti remarque que les pères éduquent " en enseignant d'abord avec l'exemple d'eux-mêmes ${ }^{64}$ ». Semblablement, Palmieri prévient que

le principal souci des pères doit être de rendre leurs enfants bons. C'est pourquoi ils doivent toujours donner de bons exemples. Car les mauvaises mœurs de la maison corrompent beaucoup plus la famille que ne le font celles du dehors, et chaque père doit être attentif et prendre garde que ses vices ne soient pas vus par ses enfants, afin que l'exemple paternel ne les dispose pas et ne les encourage pas à faire de même ${ }^{65}$.

Or, en montrant l'exemple à son élève, le précepteur acquiert une autorité naturelle qui est justement de l'ordre de la paternité, même s'il s'agit d'une paternité spirituelle et non charnelle : « considère [tes maitres] comme les parents de ton esprit et non de ton corps ", lance Piccolomini à Ladislas ${ }^{66}$. Il fait ainsi écho à Quintilien :

Je n'ai, pour le moment, qu'un seul conseil à donner aux élèves : c'est d'aimer leurs professeurs autant que les études elles-mêmes et de voir en eux des pères, non au sens physique, mais au sens intellectuel ${ }^{67}$.

6I. H.-I. Marrou, Histoire de l'éducation..., ouvr. cité, p. 33.

62. D. Lett, “"Tendres souverains”. Historiographie et histoire des pères au Moyen Âge » dans J. Delumeau et D. Roche (dir.), Histoire des Pères et de la Paternité, Paris, Larousse / HER, 2000, p. 26-30 ; P. Gavitt, Charity and Children in Renaissance Florence. The Ospedale degli Innocenti, I4I0-I536, Ann Arbor, The University of Michigan Press, 1990, p. 277-284. - Sur la répartition des tâches dans le couple, S. Vecchio, "La bonne épouse " dans G. Duby et M. Perrot (dir.), Histoire des femmes. Le Moyen Âge, C. Klapisch-Zuber (dir.), Paris, Plon, I99I, p. I4I-I45.

63. Dante, Il Convivio, G. Busnelli et G. Vandelli (éd.), Florence, Felice Le Monnier, I954, vol. 2, IV, XXIV, I4-I5 : "Vedemo ciascuno figlio più mirare a le vestigie de li paterni piedi che a l'altre. "; " [...] non li dea di sè essemplo ne l'opera, che sia contraria a le parole de la correzione. "

64. L. B. Alberti, I Libri..., ouvr. cité, p. 72 : " [...] prima con essemplo di sé stessi insegnando."

65. M. Palmieri, Vita civile..., ouvr. cité, p. 160: " [...] la principale cura de’ padri vuole essere in fare i figliuoli buoni. Per questo sempre dieno loro buoni exempli. Però che i costumi tristi di casa molto più corrompono la famiglia che non fanno quegli di fuori, et ciascuno padre debbe essere cauto et guardare i suoi vitii non sieno palesi a' figliuoli, acciò che l'exemplo paterno non gli disponga et asicuri a seguire quel medesimo. "

66. Tractatus, p. $208:$ : [...] et parentes esse non quidem corporis sed mentis tue iudicabis. "

67. Quintilien, Institution oratoire..., ouvr. cité, t. 2, II, 9, I. Voir aussi ibid., t. 2, II, 2, 4 : « Plura de officiis docentium locutus discipulos id unum interim moneo, tu preceptores suos non minus quam ipsa studia ament et parentes esse non quidem corporum, sed mentium credant. " 
Ce précepteur qui, tel un père, est un guide pour son jeune élève, est aussi un modèle pour Ladislas parce que l'imitation, chez les enfants, est spontanée. Déjà Aristote observait que "dès l'enfance, les hommes sont naturellement enclins à imiter ${ }^{68}$ ". Dans cette lignée Piccolomini recommande, également à la suite de Plutarque ${ }^{69}$, que l'entourage du puer soit à la fois exempt de tout vice et pétri de vertus :

Que ceux qui t'entourent soient emplis de bonnes mœurs, qu'il n'y ait aucun vice en eux, qu'ils n'emploient pas de mots dépravés; car nous imitons facilement tout ce qui est infâme et mauvais ${ }^{70}$.

Parfois, Piccolomini propose directement à Ladislas des modèles à imiter, sans passer par l'entremise des précepteurs. Il vante par exemple au jeune prince les mérites de certaines personnes de son entourage, tel son cousin et tuteur l'empereur Frédéric III, qu'il cite pour sa sobriété dans sa consommation de vin et de nourriture (Tractatus, p. 2I2 et p. 2I4). Piccolomini évoque également des personnalités historiques, comme Pythagore et Pompée, dont le comportement avisé vis-à-vis des aliments montre qu'il faut se nourrir non par gourmandise mais par nécessité (Tractatus, p. 2I8 et p. 220). Quant à la figure de Socrate, qui refusait de se prononcer sur le bonheur du roi des Perses parce que, disait-il, il n'en connaissait pas la vertu, elle permet à Piccolomini d'insister sur la valeur suprême qu'est l'éducation de l'intelligence, "institutio mentis».

Comme l'avait expliqué Plutarque en son temps ${ }^{71}$, l'éducation est en effet pour Piccolomini le seul bien inaliénable et nécessaire, qui appartient de façon intrinsèque à l'individu et qui ne risque pas d'être altéré par le sort, le temps ou la guerre (Tractatus, p. 220) ${ }^{72}$. Le futur pape conclut ces observations sur une mise en garde directement adressée à Ladislas, qui devra se souvenir, lui qui un jour sera très riche, que sa seule richesse est la vertu et non les biens de ce monde :

68. Aristote, Poétique, B. Gernez (éd. et trad.), Paris, Les Belles Lettres, 1997, p. II.

69. Plutarque, De l'éducation des enfants..., ouvr. cité, p. 40. On trouve la même recommandation chez Palmieri par exemple : "Sopra ogni cosa sieno nel maestro approvati costumi, però che giovando alla doctrina et nocendo al bene vivere sare' contro allo intendimento nostro che sempre propognamo l'honestamente vivere all'optimamente imparare.» (M. Palmieri, Vita civile..., ouvr. cité, p. 26.)

70. Tractatus, p. 230 : «Sint ergo, qui tibi assistunt, bonis moribus instituti, nullum sit in eis vitium, non utantur vocibus depravatis; faciles enim imitandis turpibus ac pravis omnes sumus. "

71. Plutarque, De l'éducation des enfants..., ouvr. cité, p. 43-44.

72. Lépître que Piccolomini avait adressée au duc Sigismond était déjà articulée sur le constat que le principat étant soumis aux aléas de la fortune, seule l'instruction constitue un repère solide et fiable conduisant au bien. Voir L. Guerrini, «Sulla strada della ragione... », art. cité, p. I5I-I52. 
Reçois ces mots et souviens-t'en, roi Ladislas, toi qui seras très riche un jour. Bien que tu sois appelé à un très grand règne, on ne pourra toutefois pas dire de toi que tu es heureux si tu n'abondes pas en vertu et en biens plus spirituels que matériels ${ }^{73}$.

Le fait que Piccolomini s'adresse ici directement au prince, par le vocatif, sans donner de recommandations aux précepteurs, est symptomatique de l'importance que le futur pape accorde à ses exhortations à une simplicité toute chrétienne.

Semblablement, Piccolomini s'adresse à Ladislas et non aux maîtres de ce dernier lorsqu'il aborde la dimension chrétienne de l'éducation du prince. De plus, sur cette question précise, Piccolomini ne s'exprime plus par recommandations ou par préceptes, mais par affirmations de vérités déjà constituées sur la perspective d'une autre vie après la mort ${ }^{74}$. Aussi les directives de Piccolomini sur ce point ne sont-elles pas de l'ordre du souhait; elles s'apparentent à des ordres, comme l'indique l'emploi de la deuxième personne du singulier du verbe "debere ", "devoir " (Tractatus, p. 228).

Le développement psychique de l'enfant à éduquer fait toutefois toujours l'objet d'une attention toute particulière. Piccolomini souligne à cet égard que Ladislas est trop jeune pour comprendre quelle vie terrestre peut et doit conduire à la vie après la mort : "C'est une chose qui pour être comprise exige des auditeurs plus âgés que toi ${ }^{75}$. " Et puisque les Saintes Écritures abordant cette question sont trop difficiles pour le jeune prince, il lira les textes des hommes savants :

En outre les Saintes Écritures nous conduisent à cette vie en nous instruisant par des voies mystérieuses, dont tu ne peux pas saisir le sens profond à cause de ton âge, mais il n'est pas hors de propos que tu t'exerces à partir d'autres livres, écrits par des hommes instruits $^{76}$.

Or selon Piccolomini les textes littéraires constituent des modèles à imiter, au même titre que les êtres vivants ${ }^{77}$. Dans cette proposition transparait en filigrane un écho de la conception humaniste formulée par Leonardo Bruni,

73. Tractatus, p. 222 : «Accipe hoc dictum et memorie commendato, Ladislae rex, olim ditissime, quamuis regna tibi maxima debentur, non tamen felix dici poteris, nisi virtute preditus et animi magis quam fortune bonis abundaueris. " 74. Ces vérités issues des Saintes Écritures sont confirmées, au dire de Piccolomini, par les textes païens de Socrate ou de Cicéron (Tractatus, p. 226), ces mêmes textes qu'un Giovanni Dominici avait résolument écarté de tout programme de lecture à destination des enfants. G. Dominici, Regola del governo..., p. I35. Sur cette question, voir A. Lanza, Polemiche e berte letterarie nella Firenze del primo Rinascimento (I375-I449), Rome, Bulzoni, I989, en particulier p. 8I-I06.

75. Tractatus, p. 228 : "Maiores etiam auditores quam ipse sis ad percipiendum res ipsa requirit."

76. Ibid., p. 228 : "Ad hanc porro vitam sacri ducunt sermones per occulta nos erudientes, ad quorum profundum sensum cum tibi per etate pervenire non licet. "

77. La poésie épique, ou les personnages des comédies et des tragédies sont, par exemple, riches d'excellents préceptes qui élèvent l'âme des pueri (Tractatus, p. 258 et p. 268). 
avec qui Piccolomini s'était lié d'amitié lors de son séjour à Florence ${ }^{78}$, selon laquelle les auteurs dialoguent avec leurs lecteurs ${ }^{79}$. De même, Bernardin de Sienne, dont Piccolomini a écouté les sermons avec un vif intérêt pendant sa jeunesse ${ }^{80}$, envisageait lui aussi la lecture, des docteurs de l'Église en particulier, comme un dialogue :

Maintenant va, lis leurs livres, celui qui te plaît le plus ou celui qui pour toi a le plus de valeur, et tu parleras avec eux et ils parleront avec toi; ils t'entendront et tu les entendras, et tu y prendras grand plaisir ${ }^{81}$.

Qu'il s'agisse des écrits des docteurs de l'Église, des humanistes contemporains, des historiens romains ou de la Bible, tous ces textes pourront procurer à Ladislas des modèles à suivre ou des exemples à éviter. Comme le dit Piccolomini de façon emblématique à propos de l'histoire,

il est très utile de connaître de nombreuses histoires et de les mettre en pratique, pour pouvoir, à partir de l'exemple d'autrui, suivre ce qui est utile et éviter ce qui est nocif ${ }^{82}$.

Le précepteur doit toutefois choisir les textes avec discernement et ne sélectionner que les grands auteurs. Ainsi les textes des poètes, théologiens et autres philosophes ne sont-ils pas tous à mettre à la portée des enfants (Tractatus, p. 268). Conformément à ce qu’a enseigné Basile de Cesarée, le puer se comportera comme les abeilles, qui fabriquent leur miel en butinant plusieurs fleurs différentes (Tractatus, p. 264 et p. 266), c'est-à-dire que l'enfant avisé choisira dans les livres les bons exemples et rejettera les mauvais ${ }^{83}$. C'est que, comme l'ont montré depuis longtemps les travaux d'Eugenio Garin, l'imitation humaniste n'est pas reproduction exacte d'un modèle,

78. G. Paparelli, Enea Silvio Piccolomini..., ouvr. cité, p. 27.

79. C. Bec, « De Pétrarque à Machiavel : à propos d'un topos humaniste (le dialogue lecteur/ livre) ", Rinascimento, XVI, I976, p. 3-I7.

80. Au point que Piccolomini a envisagé de suivre Bernardin, selon ses propres termes : « [...] il m’a ému au point qu'il s'en est fallu de peu que j'entre dans son ordre. Mais les supplications de mes amis m'en ont tenu éloigné. » (" [...] me intantum commouit, ut paululum abfuerit, quin et ego religionem suam ingrederer. Sed amicorum preces me retraxerunt. ") (De Bernadino Senensi) Dans Enee Silvii Piccolominei postea Pii P P. II De Viris illustribus, A. Van Heck (éd.), Cité du Vatican, Biblioteca Apostolica Vaticana, 1991, p. 38. Je remercie Serge Stolf de m'avoir indiqué cette référence. - Sur les liens entre Piccolomini et Bernardin, C.-E. Naville, Enea Silvio Piccolomini..., ouvr. cité p. 29 et p. 58-59 ; G. Paparelli, Enea Silvio Piccolomini..., ouvr. cité, p. 26.

8I. Bernardino da Siena, Le Prediche volgari edite dal padre Ciro Cannarozzi, Florence, Libreria Editrice Fiorentina, 1940, XVII, vol. 3, p. 305 : "Or va', leggi i loro libri, qual più ti piace, o di qual più fai istima, e parlerai con loro ed eglino parleranno teco; udiranno te e tu udirai loro, e gran diletto ne piglierai. "

82. Tractatus, p. 27I : "Utilissimum ergo est historias quamplures nosse atque in his se exercere, ut aliorum exemplo vet utilia sequi vel noxia vitare scias."

83. Sur cette métaphore de l'abeille, voir les commentaires de M. Ballarini et F. Buzzi, «La formazione filosofico-politica... ", art. cité, p. 578-579. Elle a été reprise entre autres par Pétrarque. Voir E. Garin, Il pensiero pedagogico..., ouvr. cité, p. $2 \mathrm{I}$. 
mais création nouvelle à partir de ce qui existe déjà ${ }^{84}$. Pour Piccolomini comme pour les humanistes, l'imitation est donc rencontre, confrontation, puis éclosion des capacités.

Cette formation que recommande Piccolomini, tout entière articulée autour de l'exemple et de l'imitation, ne peut fonctionner que si elle fait appel à la persuasion plutôt qu'à la coercition, à la récompense plutôt qu'à la sanction, à la discussion plutôt qu'aux châtiments corporels. C'est pourquoi Piccolomini recommande que les précepteurs " agissent à l'aide d'avertissements et non de $\operatorname{coups}^{85} "$, les louanges et les reproches étant selon lui plus efficaces que la violence physique. Sur ce point encore, le futur pape Pie II s'inscrit pleinement dans la tradition humaniste ${ }^{86}$.

Par exemple, si Alberti reconnaît qu'il faut savoir punir, y compris avec le fouet, "la scopa», il estime tout de même préférable de prévenir les écarts de conduite et de châtier les enfants "plutôt par la peur que par les coups ${ }^{87}$ ". Guarino Veronese compte davantage sur «l'exemple vivant et [...] l'autorité " que sur la "sévérité des coups ${ }^{88}$ ". Quant à Palmieri, il concède que les enfants peu aptes à la vertu et destinés aux arts mécaniques méritent parfois qu'on les frappe, mais il s'attache en revanche longuement à démontrer que les coups sont inutiles, voire néfastes. Car non seulement les mises en garde peuvent suffire, mais une punition suivie d'une réflexion sur la faute commise est plus efficace qu'une douleur qui n'est que physique et éphémère ${ }^{89}$. De plus, la sanction corporelle est selon Palmieri « destinée à rendre les âmes esclaves $^{90}$ ", formulation qui est employée également par Maffeo Vegio : "Que les parents soient plus attentifs lorsqu'ils corrigent leurs enfants; car la correction convient aux esclaves et non aux hommes libres ${ }^{91}$. "

Cette idée, héritée de Plutarque ${ }^{92}$ et de Quintilien ${ }^{93}$, selon laquelle les châtiments physiques conviennent aux hommes privés de liberté, figure

84. E. Garin, L'éducation de l'homme moderne, I400-1600, Paris, Fayard, p. 99-105.

85. Tractatus, p. $206:$ : [...] monitis, non plagis agant."

86. Il se distingue au contraire d'un Dominici, qui explique à propos des jeunes gens que " toujours et en toutes circonstances les coups et les corrections leur sont utiles, et ils s'y habitueraient s'ils aimaient Dieu et non la chair. " (" [...] sempre e in ogni caso sono loro utili le busse e battiture, e a questo s'avvezzerebbono se s'amassono in Dio, e non pure alla carne ", G. Dominici, Regola del governo..., ouvr. cité, p. 204.)

87. L. B. Alberti, I Libri..., ouvr. cité, p. 70-72 : " [...] più tosto con paura che con busse."

88. "l'esempio di vita e [...] l'autorità ", "severità di busse ", Lettera di Guarino Veronese al suo carissimo Martino dans E. Garin, Il pensiero pedagogico..., ouvr. cité, p. 355.

89. M. Palmieri, Vita civile..., ouvr. cité, p. 36.

90. Ibid., p. $36:$ : [...] atta a fare gli animi servi."

91. Maffeo Vegio da Lodi, De educatione liberorum et eorum claris moribus dans E. Garin, Il pensiero pedagogico..., ouvr. cité, p. I78 : "Quare cautius versentur parentes in emendatione fliorum; servis enim ea, non liberis hominibus conveniunt."

92. Plutarque, De l'éducation des enfants..., ouvr. cité, p. 5I.

93. Quintilien, Institution oratoire..., ouvr. cité, t. I, I, 2, I4. 
aussi dans le Tractatus de liberorum educatione de Piccolomini : " [...] les coups de verges servent aux esclaves et non aux hommes libres ${ }^{94}$. " Il est d'ailleurs connu que les humanistes cherchent avant tout à faire émerger les dispositions naturelles propres à l'enfant en respectant sa personnalitéps. L'éducation est donc avant tout un modelage. Il s'agit de façonner le puer selon un idéal à atteindre, en tenant compte de ses goûts, de ses aspirations et de ses capacités, et non de le contraindre à obéir par la force physique ${ }^{96}$.

Le but de l'éducation consiste dès lors moins à encadrer le développement de l'enfant tel qu'il est aujourd'hui, qu'à préparer l'homme qu'il sera demain ${ }^{97}$; ce n'est pas un hasard si Piccolomini fait justement de multiples allusions à l'avenir de Ladislas et s'il souligne que l'acquisition des vertus au cours de l'enfance permet de préparer la vieillesse (Tractatus, p. 222).

Dans cette perspective, la persuasion, à travers « les avertissements et les raisonnements ", "monitus ac rationibus", écrit Piccolomini, fait davantage que la violence des coups. Car la brutalité suscite la haine, qui est particulièrement nuisible à l'apprentissage. Un dosage équilibré des reproches qui détournent du mal d'un côté, et des félicitations qui encouragent au bien de l'autre, est par conséquent la seule pédagogie possible.

L'analyse qui précède montre que pour Piccolomini comme pour les humanistes, la parole est l'instrument le plus adapté et le plus efficace pour éduquer les enfants. Mais la parole n'est pas seulement un moyen de formation. La parole est aussi source de connaissance, qu'il s'agisse des recommandations que les précepteurs adressent à leur élève ou des textes que le puer découvre. De plus, l'enseignement que l'élève reçoit à travers l'étude des textes est double. D'une part, il apprend à s'exprimer correctement, élégamment et honnêtement, et d'autre part, il acquiert la vertu indispensable à l'homme qui veut gouverner :

Ainsi tu tireras un double avantage de la lecture des auteurs anciens et modernes qui écrivirent avec sagacité. Car tu obtiendras une vie meilleure grâce à l'étude de la vertu,

94. Tractatus, p. $206:$ "[...] verbera servos decent, non liberos."

95. Voir par exemple M. Palmieri, Vita civile..., ouvr. cité, p. 48-49 ; P. P. Vergerio, De ingenuis moribus et liberalibus studiis adulescentie dans E. Garin, Il pensiero pedagogico..., ouvr. cité, p. I28-I29 et Maffeo Vegio da Lodi, De educatione liberorum..., ouvr. cité, p. 192-193. Même le très sévère Dominici remarque qu'il est inutile, voire contre-productif, d'aller à l'encontre des préférences des enfants. G. Dominici, Regola del governo..., ouvr. cité, p. 2I4. Quintilien, pour ne citer que lui, formulait la même mise en garde (Quintilien, Institution oratoire..., ouvr. cité, t. 2, II,8,6).

96. P. Gavitt, Charity and Children..., ouvr. cité, p. 273 et p. 284 ; E. Becchi, " Humanisme et Renaissance " dans E. Becchi et D. Julia (dir.), Histoire de l'enfance..., ouvr. cité, p. I8I-I82.

97. H.-I. Marrou, Histoire de l'éducation..., ouvr. cité, p. 299. 
et tu te procureras une grande habileté pour la grammaire et l'art du discours et une grande réserve de mots et de sentences remarquables et très élégants ${ }^{98}$.

C'est pourquoi le langage remplit une fonction fondamentale dans l'éducation du prince à l'art de gouverner.

Un terme pourrait en effet parfaitement résumer la pédagogie politique de Piccolomini. Il s'agit du mot " langage », entendu au sens large et courant du terme, c'est-à-dire le " langage " comme la possibilité humaine d'exprimer une pensée et de la communiquer. Comme le remarque Lionello Sozzi, Piccolomini indique que le langage est la faculté la plus élevée de l'homme, une faculté qui le distingue des bêtes ${ }^{99}$ : la dignité de l'homme est inséparable de sa capacité à s'exprimer - et c'est là un point de vue qu'Enea Silvio partage une fois de plus avec les humanistes ${ }^{100}$. Mais surtout, lorsqu'on cherche un dénominateur commun aux recommandations que Piccolomini fait à Ladislas, on trouve systématiquement le langage, un langage qui permet la communication entre le prince et ses sujets.

Le langage du prince a tout d'abord une dimension corporelle. Plus exactement, le mouvement du corps princier doit refléter et incarner l'esprit noble que le souverain a reçu à la naissance : « il faut faire en sorte que tes gestes correspondent à ta beautéror ", explique Piccolomini, en rappelant à la suite de Quintilien que c'est d'ailleurs pour cette raison que les Grecs avaient conçu la "chironomie ", cet art d'adapter le geste des mains au discours ${ }^{102}$. C'est donc par son corps que le prince dit qui il est et qu'il manifeste sa grâce et sa puissance à ses sujets. Voilà pourquoi Piccolomini détaille les moindres attitudes corporelles de Ladislas, jusqu'au mouvement de ses cils qu'il doit maîtriser, parce que « tout ce qui n'est pas convenable ne peut pas plaire ${ }^{\mathrm{IO}}$ ”.

Destiné à gouverner, Ladislas doit avant tout se préoccuper de l'effet que son attitude provoquera sur ses sujets - et sur ce point, on pourrait voir ici une anticipation de la formulation de Machiavel qui cherchera la «vérité

98. Tractatus, p. 266 : «Sic ex lectione veterum et novorum qui scripserunt prudenter auctorum duplex te utilitas comitabitur. Nam et vitam studio virtutum efficies meliorem, et grammaticam peritiamque sermonis optimorum et ornatissimorum verborum ac sententiarum magnam tibi suppellectilem comparabis. "

99. L. Sozzi, « La dignitas hominis nel pensiero di Pio II »..., art. cité, p. 53.

Ioo. A. Musumeci, "L'epistolario di Enea Silvio Piccolomini. Il discorso sulla letteratura » dans Pio II e la cultura..., p. 373 .

IOI. Tractatus, p. $208:$ : [...] studendum est ut gestus forme respondeant. "

IO2. Quintilien, Institution oratoire..., ouvr. cité, t. I, I,II,I7.

I03. Tractatus, p. 208 : " [...] nihil potest placere quod non decet. " Voir Quintilien, Institution oratoire..., ouvr. cité, t. 6, XI, 3, 72-79. 
effective des choses ${ }^{104}$ ». En tout cas cet intérêt pour l'effet explique pourquoi Piccolomini s'attarde longuement sur l'image sociale du puer qu'il veut éduquer. Emblématique à cet égard est le passage du Tractatus de liberorum educatione où Piccolomini recommande à Ladislas de ne pas commettre d'excès de coquetterie vestimentaire. Alors que Dominici, par exemple, voit dans le superflu un obstacle à la piété des enfants ${ }^{\mathrm{IO}}$, Piccolomini estime qu'il serait honteux qu'un roi apparaisse efféminé aux yeux de ses sujets ${ }^{106}$. Là où Dominici fait de cette question un enjeu qui relève de la vie privée et spirituelle, Piccolomini y voit donc au contraire une question d'ordre politique qui relève de la vie publique du roi. Significativement, Piccolomini souligne ensuite que l'équilibre entre coquetterie et sobriété est d'autant plus difficile à atteindre pour un roi qu'il risque d'être taxé d'avarice, accusation encore plus nuisible à sa réputation que celle de vanité (Tractatus, p. 220) : il s'agit bien là de l'image que le souverain renvoie à ses sujets!

De même, lorsque Piccolomini invite Ladislas à boire du vin de façon modérée, ce n'est pas parce que l'alcool pourrait causer un quelconque dommage physiologique à son corps, mais parce que l'image qu'un enfant ivre donne de lui-même est répugnante : " [...] il n'y a rien de plus honteux que le goût d'un enfant pour le vin ${ }^{107}$. " Pourtant, les dommages causés par l'alcoolisme étaient connus depuis l'Antiquité. Des recherches publiées par l'Association Guillaume Budé ont ainsi montré que Sénèque et Pline l'Ancien mentionnaient « les altérations de la morphologie, les perturbations locomotrices, les perturbations sensorielles [...] la pathologie articulaire ${ }^{108}$ " dues à l'absorption de vin. D'ailleurs le vin était interdit à la consommation avant l'âge de vingt-et-un ans, conformément aux recommandations de Galien pour qui il ne fallait pas attiser le feu de la jeunesse ${ }^{\text {109. }}$. Si un Maffeo Vegio reprend cette idée pour priver les enfants de vin ${ }^{\text {IIO }}$, Piccolomini se préoccupe en revanche surtout du message que le prince enverrait à ses

I04. N. Machiavelli, De principatibus - Le Prince, G. Inglese (éd.), J.-C. Zancarini et J.-L. Fournel (trad.), Paris, PUF, 2000, XV : «verità effettuale delle cose ».

I05. G. Dominici, Regola del governo..., ouvr. cité, p. 196.

Io6. Tractatus, p. 220. Chez Palmieri aussi l'apparence vestimentaire est une affaire de virilité (M. Palmieri, Vita civile..., ouvr. cité, p. 36-37).

I07. Tractatus, p. 216 : «Appetitore vini puero nibil turpius est.»

I08. J.-M. André, «Vertu thérapeutique du vin et pathologie de l'ivresse à Rome, de Lucrèce à Pline l'ancien » dans Les écrivains et le sacré. La vigne et le vin dans la littérature (association Guillaume Budé, actes du XII ${ }^{e}$ congrès, 1988), Paris, Les Belles Lettres, I989, p. 45I.

I09. A. Pazzini, «Il vino nella storia, nel costume, nella medicina (con particolare riguardo all'epoca romana) » dans Pagine di storia della medicina, 1971, vol. I5, p. 23.

IIo. Maffeo Vegio cite Platon et Sénèque : «Cependant, parce qu'ils abondent de chaleur, il ne faut pas du tout leur donner de vin; [...] comme prévient Platon en interdisant d'exciter le feu avec le feu. " (Vinum autem, quia calore abundant, minime eis ministrabitur, quod [...] Plato monet vetans ignem igni excitari." Dans Maffeo Vegio da Lodi, De educatione liberorum..., ouvr. cité, p. 175.) 
sujets s'il se montrait ivre. Ainsi souligne-t-il que l'excès de vin (tout comme l'excès de nourriture d'ailleurs) pourrait altérer toutes les facultés intellectuelles et en particulier les capacités à gouverner du prince. Car chez l'enfant élevé dans l'ivresse, «il n'y a pas de mémoire, il n'y a pas de vivacité d'esprit, il n'y a pas de désir pour les beaux-arts, pour la gloire, pour la vertu ${ }^{\mathrm{III}}{ }^{\prime}$.

Le prince ne communique toutefois pas uniquement avec son corps; il s'adresse à ses sujets également par la parole, qu'elle soit orale ou écrite.

De la même façon qu'on éduque un enfant plus par la parole que par la force, Piccolomini considère que l'amour est aussi important que la force pour gouverner un peuple; c'est pourquoi il cherche à faciliter la communication entre Ladislas et ses sujets. Pour commencer, le jeune prince doit pouvoir parler directement à la population, sans avoir besoin de recourir à un interprète (Tractatus, p. 232); il en va ici de sa propre liberté, car un prince qui ne pourrait pas s'exprimer de lui-même serait plus sujet que souverain. Il devra donc s'entourer de jeunes gens s'exprimant dans la langue parlée en Hongrie et en Bohème (Tractatus, p. 233), afin d'apprendre à comprendre ses sujets et à s'adresser à eux. Notons qu'il s'agit là d'une pédagogie empruntée à la tradition médiévale, où le jeune prince était souvent entouré d'une troupe d'enfants chargés de sa socialisation ${ }^{\mathrm{II} 2}$.

En outre les qualités oratoires sont indispensables au prince s'il veut gagner les faveurs de la population qu'il gouverne : "Rien ne procure davantage la faveur du peuple à son prince que la grâce de son discours ${ }^{\mathrm{II}}$. » Aussi doit-il être formé dès la pueritia à s'exprimer avec correction et sagesse. La correction du langage inclut aussi bien le respect de la syntaxe, de la morphologie et du sens des mots (Tractatus, p. 240 et p. 246) que la qualité de la diction et du timbre de la voix, qui ne devra être ni efféminé, ni chevrotant, ni tonitruant (p. 236). Quant à la sagesse du langage, elle dépend de l'équilibre entre le silence et la parole (p. 234) que recommandait déjà Plutarque ${ }^{\mathrm{II}}$, de la sincérité du discours et du respect qu'il manifeste visà-vis des auditeurs (p. 236), enfin de son intelligibilité.

Car il importe avant tout que le prince soit parfaitement compris. C'est pourquoi l'habitude, la consuetudo, sera le critère absolu de choix des mots (Tractatus, p. 256), à condition bien entendu que le prince adopte les habitudes de langage des personnes cultivées et non de celles qui donnent le mauvais exemple (p. 258). S'il s'agit là d'une recommandation déjà présente

III. Tractatus, p. 216 : "Non ibi memoria est, non vivax ingenium, non bonarum cupido artium, non glorie, non honestatis studium."

II2. E. Becchi, «Le Moyen Âge », E. Becchi et D. Julia (dir.), Histoire de l'enfance..., p. I22-I23.

II3. Tractatus, p. 232 : "Nihil est, quod suo principi favorem populi magis conciliet, quam gratia sermonis. "

II4. Plutarque, De l'éducation des enfants..., ouvr. cité, p. 55. 
chez Quintilien IIs, elle est chez Piccolomini emblématique de l'importance qu'il confère à la communication entre le prince et ses sujets.

Significatives à cet égard sont les règles qui président au choix des mots : si le prince doit choisir entre deux synonymes, il prendra le mot qui se comprend le plus facilement et le mot le plus beau. Piccolomini mesure donc ici les effets positifs qu'auront sur les sujets l'intelligibilité et l'esthétique du discours du prince. C'est qu'il envisage le lien entre le souverain qui gouverne et les sujets qui obéissent comme une relation d'échange, comme le montre la comparaison, empruntée à Quintilien, qu'il opère entre le langage et la monnaie : "Il faut employer le discours comme la monnaie, dont le coin est public ${ }^{\mathrm{II}}$. " Le langage, parce qu'il constitue le lien fondamental qui unit le prince et son peuple, doit ainsi être connu de tous, il doit être un bien à partager.

En d'autres termes, bien parler, c'est bien gouverner, tout comme bien gouverner consiste à bien parler. Déjà dans une lettre adressée à Adam des Molins en I444, Piccolomini affirmait que l'éloquence assure le succès de l'homme en politique :

Il n'y a rien qui n'assure autant le cours des affaires que l'éloquence, parce que, quoi qu'on fasse dans les affaires publiques, on utilise des mots persuasifs dont l'idée reste dans le peuple, ce qui peut le mieux le convaincre ${ }^{117}$.

Dans le Tractatus de liberorum educatione, cette idée prend la forme de la célèbre phrase empruntée à Démocrite, selon laquelle " la parole est l'ombre de l'action" (" actionis umbra [est]", Tractatus, p. 236).

Cette observation vaut également pour le langage écrit qui fonde le lien entre le prince et ses sujets au même titre que le langage oral. C'est pourquoi la grammaire occupe dans le Tractatus une place écrasante par rapport aux matières du quadrivium et même aux deux autres matières du trivium. Si Piccolomini inclut ces disciplines dans le programme de formation qu'il destine à Ladislas, il le fait de façon tout à fait secondaire. Car la grammaire comprend, comme chez Quintilien, la scientia recte loquendi, la ratio scribendi et l'enarratio poetarum.

II5. «Donc, pour le langage, j’appellerai “usage” l'accord des gens cultivés, comme, pour la vie, celui des honnêtes gens. " ("Ergo consuetudinem sermonis uocabo consensum eruditorum, sicut uiuendi consensum bonorum. " Dans Quintilien, Institution oratoire..., ouvr. cité, t. I, I, 6,45.)

II6. Tractatus, p. 256 : «Utendum est sermone ut nummo, cui publica forma est. ». Voici ce qu'en dit Quintilien : "L'usage, toutefois, est le maître le plus sûr du parler et on doit traiter le langage comme une monnaie qui est marquée au coin de l'État. " ("Consuetudo uero certissimo loquenti magistra, utendumque plane sermone, ut nummo, cui publica forma est. " Dans Quintilien, Institution oratoire..., ouvr. cité, t. I, I, 6, 3.)

II7. A. Musumeci, "L'epistolario di Enea Silvio Piccolomini..." ", art. cité, p. 382 : "Nichil est, quod tam regat orbem quam eloquentia, quoniam, quicquid agimus in re publica, persuasi uerbis agimus et illius in populis sententia manet, qui melius nouit persuadere. " 
Pour travailler l'orthographe ${ }^{\text {II8 }}$, le prince fera porter ses efforts aussi bien sur la calligraphie que sur la correction grammaticale. Ce dernier point se fondera, comme pour le langage parlé, sur la consuetudo transmise par les meilleurs auteurs (Tractatus, p. 280 et p. 282). Les exercices que Ladislas effectuera en ce sens lui permettront d'ailleurs de s'imprégner de textes édifiants lorsqu'il les recopiera (p. 274). Par ailleurs, de la même façon que le roi doit éviter de s'appuyer sur des interprètes pour communiquer avec ses sujets, il écrira ses textes lui-même, sans se reposer sur ses seuls scribes. Et comme il est préférable qu'un roi écrive en faisant apparaitre des "perles " plutôt que des "chiures de mouches " ${ }^{\text {In }}$, il a tout intérêt à travailler l'art du discours. Pour ce faire il puisera les règles grammaticales chez les orateurs mais apprendra l'usage de la rhétorique chez les poètes et les historiens (p. 284).

Le langage a donc plusieurs fonctions chez Piccolomini. Instrument de formation (les précepteurs éduquent leurs élèves par la parole), moyen de communication (le roi s'adresse à ses sujets par la parole), il est aussi source de connaissance (le prince se forme à l'éloquence par les textes littéraires).

Bien plus, le langage des textes est nécessaire pour former le prince à l'art de gouverner. Voici ce qu'Enea Silvio dit des études littéraires : « [...] elles rapportent le passé, elles règlent le présent, elles indiquent le futur ${ }^{120}$. Cette formulation, qui s'articule autour de la combinaison de la mémoire, de l'intelligence et de la prévoyance constitue la définition même de la " prudence ». Le Repertorium morale, une des encyclopédies les plus consultées au Moyen Âge, indiquait en effet que la prudence consiste "dans la mémoire du passé, la mise en ordre du présent et la méditation du futur ${ }^{\mathrm{I2I}}$ ». À son tour Dante, reprenant cette définition héritée des Romains, soulignait que l'homme prudent sait combiner le passé et le présent pour prévoir le futur :

[...] il convient [...] d'être prudent [...] : et pour cela il faut une bonne mémoire des choses passées, une bonne connaissance des choses présentes et une bonne prévoyance des choses futures ${ }^{122}$.

II8. Voir sur ce point U. Pizzani, «La sezione ortografica del De liberorum educatione di Enea Silvio Piccolomini » dans L'educazione e la formazione intellettuale..., ouvr. cité, p. I77-I85.

II9. Tractatus, p. 272 : "margaritas", "muscarum lituras".

I20. Ibid., p. $224:$ : [...] preterita referunt, presentia moderantur, futura indicant."

I2I. E. Panofsky, L'œuvre d'art et ses significations. Essais sur les arts visuels, Paris, Gallimard, 1969, 258-262.

I22. Dante, Il Convivio..., ouvr. cité, IV, XXVII, $5:$ : [...] conviensi [...] essere prudente [...] : e a ciò essere si richiede buona memoria de le vedute cose, buona conoscenza de le presenti e buona provedenza de le future." — Voir à ce propos Y. Lehmann, "Prudentia chez les penseurs romains. Essai d'investigation philosophique et morale " dans La représentation de la prudence (actes du Colloque de l'université de Haute Alsace, 5 mars I999), Chroniques italiennes, $\mathrm{n}^{\circ} 60$, avril 1999, p. I3-19. 
Encore plus significatif pour l'interprétation du traité qui nous occupe, Bernardin de Sienne, dont on a déjà souligné l'influence sur Piccolomini, rappelait dans un de ses sermons prononcés en 1425 , que « la prudence se souvient du passé, ordonne le présent et pourvoit au futur ${ }^{\mathrm{r23}}$ ".

Or, la prudence est indiquée comme indispensable au souverain dès la deuxième phrase de l'épître composée par le futur pape Pie II pour Ladislas :

Le fait est que quand tu ne seras plus sous tutelle tu devras gouverner de très grands royaumes et de très vastes principautés, que tu ne pourras pas gouverner longtemps sans une prudence parfaite ${ }^{124}$.

Il s'agit ici d'une vertu morale proche de la phronésis aristotélicienne tout entière tournée vers l'action ${ }^{125}$. De fait, comme le montre Lionello Sozzi en citant un passage du Triplicis problematis resolutio de Piccolomini, la prudence, parce qu' elle enflamme l'âme et les affects, est action pure : "Donc sera prudent celui qui sait, qui veut et qui agit selon les bonnes choses $^{126}$."

Mais les contemporains de Piccolomini considèrent la prudence comme l'apanage des vieux ${ }^{\mathrm{I} 27}$. À la fin du XIv ${ }^{\mathrm{e}}$ siècle, Franco Sacchetti par exemple écrivait que «les jeunes ne peuvent pas être prudents, parce que la prudence exige l'expérience, qui a besoin de temps ${ }^{\mathrm{I2} 8}$ ». Quant à Alberti, il faisait des cheveux blancs, ces emblèmes de la vieillesse, le symbole même de la prudence : " $[\ldots]$ ces cheveux blancs me rendent prudent en toute chose ${ }^{129}$." Piccolomini lui-même, dans sa lettre à Sigismond en I443, recommandait de ne pas confier le pouvoir à des hommes jeunes et encore inexpérimentés ${ }^{130}$.

\footnotetext{
I23. Bernardino da Siena, Le prediche volgari..., ouvr. cité, XVI, p. 292 : "La prudenzia si ricorda del passato, ordina il presente e provede al futuro. "

124. Tractatus, p. 198 : "Maximis nanque regnis et amplissimis principatibus, postquam tutele finieris annos, expectaris imperaturus, quibus nisi consumate prudentie fueris, diu dominari non poteris. "

I25. P. Aubenque, La prudence chez Aristote, Paris, PUF, I986, p. 34.

I26. L. Sozzi, « La dignitas hominis nel pensiero di Pio II »..., art. cité, p. 5I : « Erit igitur prudens qui scit, vult et agit quae bona sunt."

I27. Sur ce point et l'importance de la prudence dans l'art de gouverner, voir C. Terreaux-Scotto, Les âges de la vie dans la pensée politique florentine républicaine de la révolte des Ciompi à la chute de la République, thèse de doctorat soutenue sous la direction de Jean-Louis Fournel à l'université Paris 8, 200 .

I28. "Iuvenes non possunt esse prudentes, quia prudentia exquirit experentiam, que indiget tempore ", cité par C. Bec, Les marchands écrivains. Affaires et humanisme à Florence. 1375-I434, Paris / La Haye, Mouton \& Co / École pratique des hautes études, 1967, p. 329.

I29. L. B. Alberti, I Libri..., p. 25 : " [...] questi capelli [canuti] di tutto mi fanno prudente. "

I30. M. Lentzen, « Le idee pedagogiche di Enea Silvio Piccolomini »..., art. cité, p. 585. — Voir Æneæ Sylvii Pii, Illustrissimo principi ex sanguine Cesarum fato, Domini Sigismundo Austrie dans Aenee Sylvii Piccolominei Senensis, qui post adeptum pontificatum Pius eius nominis secundus appellatus est opera qua extant omnia, nunc demum post corruptissimas aeditiones summa diligentia castigata \& in unum corpus redacta, quorum elenchum uersa pagella indicabit, Frankfurt, Minerva, 1967, p. 606.
} 
Tout se passe donc comme si les études littéraires conféraient au jeune prince la prudence qu'il n'a pas encore eu le temps d'acquérir.

En plus de la prudence, les lettres apportent la faculté de discerner le bien du mal (Tractatus, p. 224) ainsi que la liberté de ne pas se laisser séduire par les discours des adulateurs (p. 224). Les études littéraires constituent dès lors une formation destinée à donner au prince la sagesse, sagesse dont Piccolomini affirme que, comme la prudence, elle est absolument nécessaire pour gouverner : "Personne n'a besoin de la sagesse autant que ceux qui gouvernent ${ }^{131}$."

Mais cette sagesse est avant tout faite d'action. Car en aucun cas la culture, ou la "philosophie ", selon le terme employé par Piccolomini, n'est une fin en soi. Bien au contraire, dans une perspective très utilitaire $^{132}$, il considère la philosophie comme un moyen pour atteindre un but : elle enseigne au prince à communiquer avec ses sujets. La philosophie est dès lors le fondement de tout engagement dans la res publica (Tractatus, p. 200). Elle constitue même le lien qui unit le roi à la population qu'il gouverne. Piccolomini, s'appuyant en cela sur les réflexions de Plutarque, explique en effet que non seulement la philosophie apprend au souverain à vénérer Dieu, mais qu'elle lui apprend aussi à se comporter avec chacune des catégories qui composent l'ensemble de ses sujets, des soldats aux serviteurs en passant par les magistrats et les étrangers. La philosophie permet en outre au roi de respecter les femmes, de montrer sa tendresse envers sa famille, de ne pas maltraiter ses serviteurs, d'honorer les plus âgés, d'obéir aux lois, de ne céder ni à la colère ni aux plaisirs, enfin de récompenser les bons et de punir les mauvais ${ }^{133}$.

Loin d'être un substitut à la vie sociale, les lettres en sont donc au contraire le fondement et le ciment ${ }^{134}$. C'est pourquoi il ne s'agit pas pour le prince de séparer la vie contemplative de la vie active, mais de trouver le juste équilibre entre les deux, ou, encore mieux, de savoir mettre la première au service de la seconde, en d'autres termes, de savoir utiliser les connaissances transmises par la culture pour gouverner son peuple. Là se trouve la perfection; Piccolomini écrit qu'

\footnotetext{
131. Tractatus, p. 199 : "Nulli magis quam regnanti opus est sapientia. "

I32. A. Musumeci, "L'epistolario di Enea Silvio Piccolomini... ", art. cité, p. 379.

133. Tractatus, p. 294. On trouve exactement les mêmes remarques dans le traité de Maffeo Vegio, mais appliquées à l'ensemble des jeunes gens. Maffeo Vegio da Lodi, De educatione liberorum..., ouvr. cité, p. 196-197. C'est que la source, commune, se trouve chez Plutarque (De l'éducation des enfants..., ouvr. cité, p. 48).

134. Dans la lettre adressée en I443 à Sigismond d'Autriche, Piccolomini avait déjà souligné que la littérature n'est pas une fin en soi et qu'elle ne doit pas entraver le développement de la vie sociale (voir A. Musumeci, "L'epistolario di Enea Silvio Piccolomini... ", art. cité, p. 38I).
} 
en effet seuls sont parfaits les hommes qui peuvent mêler les actions civiles à la philosophie et revendiquer pour eux-mêmes ces deux bonnes choses ${ }^{135}$.

Certes, il s'agit là encore d'une idée héritée de Plutarque ${ }^{\mathrm{I3} 6}$ chère aux humanistes, aux humanistes civils en particulier ${ }^{137}$. Alberti remarque de façon emblématique qu'

il serait long de dire combien les lettres sont, je ne dis pas utiles, mais nécessaires pour ceux qui dirigent et gouvernent les choses; et je ne décris pas non plus quel ornement elles sont pour la République ${ }^{138}$.

De plus, l'affirmation de Piccolomini selon laquelle un programme pédagogique articulé autour des études littéraires confere la vertu au prince ${ }^{139}$ pourrait s'appliquer à n'importe quel souverain. Ce n'est pas un hasard si le futur pape reprend à ce propos la formulation de Jean de Salisbury pour lequel un roi ignare est un "âne couronné ", "coronatum asinum ${ }^{\mathrm{I} 40}$ ". L'absence d'instruction fait ainsi perdre au prince toute légitimité politique. Car pour gouverner ses sujets, le prince doit savoir se comporter et parler, deux compétences qu'il acquiert grâce au savoir contenu dans les livres. De sorte que l'instruction permet de transformer la puissance héritée des ancêtres en autorité propre au prince ${ }^{\mathrm{I} 4 \mathrm{I}}$.

Mais le Tractatus de liberorum educatione a une dimension particulière. Dès les premières lignes de son épître, Enea Silvio confie une véritable mission, pour l'avenir, à Ladislas. Car si le futur pape invite le jeune prince à suivre le modèle des dirigeants de l'Antiquité, remplis de "gloire " et "d'érudition ", c'est pour qu'il redonne à la Hongrie et à la Bohème leur splendeur révolue. Ladislas apparaît ainsi comme un rédempteur capable d'effacer le passé :

Donc quand sera venu le moment de régner, je désire que tu prennes modèle sur eux, pour que la Hongrie, accablée par de grandes calamités, et la Bohème, opprimée par des erreurs hérétiques, puissent respirer et qu'elles retrouvent leur splendeur d'autrefois ${ }^{\mathrm{I} 2}$.

135. Tractatus, p. 200 : "Soli nanque perfecti sunt homines, qui civiles cum philosophia partes queunt immiscere sibique gemina vendicant bona."

136. Plutarque, De l'éducation des enfants..., ouvr. cité, p. 49.

I37. L. Guerrini, «Sulla strada della ragione... ", art. cité, p. I29-I3I.

138. L. B. Alberti, I Libri..., ouvr. cité, p. 85 : « [...] sarebbe lungo recitare quanto siano le lettere, non dico utili, ma necessarie a chi regge e governa le cose; né descrivo quanto elle siano ornamento alla repubblica."

I39. Tractatus, p. 200 : "Ad virtutem autem capessendam litterarum studia plurimum adiumenti prebent. "

I40. Ibid., p. 200. Kallendorf a bien montré que Piccolomini connaissait directement le texte de Jean de Salisbury. Voir sur ce point L. Guerrini, «Sulla strada della ragione... », art. cité, p. I76-I8o.

I4I. L. Guerrini, ibid., p. I58.

I42. Tractatus, p. 200 : "Ad horum ergo te modum, cum tempus aderit, cupimus regnaturum, ut fatigata magnis calamitatibus Hungaria ac servis hereseos erroribus conculcata Bhoemia te duce respirent splendorique pristino restituantur." 
Il faut dire que Piccolomini ne manque pas une occasion de critiquer les us et coutumes des futurs sujets de Ladislas. La population d'Autriche, de Hongrie et de Bohème s'adonne de façon excessive aux plaisirs de la table, flattant les organisateurs de banquets et se comportant en parasite (Tractatus, p. 2I2); de même, c'est l'exemple d'un vieil habitant de Bohème que prend Piccolomini pour dénoncer ceux qui incitent les enfants à boire du vin. Mais son jugement négatif ne se borne ni à l'époque contemporaine ni à des remarques concernant la vie quotidienne; il s'étend aussi au passé de ces pays, puisque d'après lui l'histoire de la Bohème et de la Hongrie n'a aucune valeur esthétique, intellectuelle ou morale, au point qu'elle ne doit pas être enseignée au puer Ladislas (p. 270). Cette posture n'a rien d'étonnant de la part de Piccolomini, qui avait par ailleurs dressé un portrait particulièrement sévère de ces pays : ivrognerie, superstition, manque d'amabilité, esprit rebelle, coutumes barbares, brigandage, hérésie, aucune critique n'est absente de son Histoire de la Bohème ${ }^{\mathrm{I} 43}$. C'est par conséquent dans l'histoire grecque et romaine et non dans l'histoire récente des pays qu'il devra diriger que Ladislas puisera des modèles de gouvernement pour être digne de sa fonction (p. 200).

Derrière les remarques générales sur l'éducation des enfants, qui ont pu faire dire à Del Donno qu' "il y a dans le prince tous les jeunes que l'humanisme éduque à la vie civile et politique ${ }^{\mathrm{I} 44}$ ", il y a donc surtout une véritable polémique contre les peuples germaniques ${ }^{\mathrm{I} 45}$.

Piccolomini, qui s'intéresse avant tout aux effets que le comportement du prince aura sur ses sujets, fait du langage le pivot de la pédagogie qu'il propose. Pour le dire avec Antonio Musumeci, "Piccolomini était convaincu de l'importance du langage comme corrélatif d'une forme intérieure : bien parler signifier bien agir - l'éloquence comme vertu ${ }^{146} »$. Mais on pourrait tout aussi bien appliquer cette observation à Piccolomini lui-même. En rédigeant cette épître, dans laquelle il confie une mission au puer Ladislas, le futur pape Pie II agit bien, c'est-à-dire qu’il cherche à éduquer la population

I43. Voir De situ regionis Bohemica, de fluminibus eiusdem \& civitatibus, ac de Bohemorum moribus dans Aenea Sylvii Piccolominei Senensis, qui post adeptum pontificatum..., ouvr. cité, p. 8I-I43 et les commentaires de J. B. Novàk, "Enea Silvio Piccolomini e la sua Storia di Boemia ", Rivista italiana di Praga, n I, janvier-juin 1927, fasc. I, p. I8-38 et en particulier p. 34-36.

144. M. Del Donno, Enea Silvio Piccolomini. L’educazione dei giovani..., ouvr. cité, p. 94 : «[...] nel principe vi sono tutti i giovani che l'umanesimo educa alla vita civile e politica. "

I45. L. Guerrini, "Sulla strada della ragione... ", art. cité, p. I65.

I46. A. Musumeci, "L'epistolario di Enea Silvio Piccolomini... ", art. cité, p. 377 : " [...] il Piccolomini era convinto dell'importanza della parola come correlativo di una forma interiore: parlar bene significa agire beneeloquenza come virtù." 
de l'Europe orientale conformément aux préceptes humanistes développés dans la péninsule dont il est originaire ${ }^{\mathrm{I} 47}$. De ce point de vue, la lettre serait alors aussi bien destinée aux futurs sujets de Ladislas qu’au prince lui-même. Piccolomini utiliserait alors le langage comme un véritable instrument politique pour soumettre les peuples germaniques aux valeurs humanistes qui sont les siennes.

Le Tractatus de liberorum educatione se présente dès lors autant comme un programme politique que comme un traité pédagogique. En formant Ladislas à accéder au trône de Hongrie et de Bohème, c'est toute une population que Piccolomini cherche à éduquer. La figure de Ladislas serait ainsi moins l'emblème de tous les enfants, comme il a souvent été dit, qu'une personnalité singulière, appelé à guider un pays dont les habitants s'étaient fourvoyés dans les chemins hérétiques. 